\title{
Comparison of the Effects of Aromatherapy with Rose and Lavender on Physiological Parameters of Patients Undergoing Open Heart Surgery: A Clinical Trial
}

\author{
Saeed Ghasemi, ${ }^{1}$ Hosien Babatabar Darzi, ${ }^{1,}$ and Abbas Ebadi ${ }^{2}$ \\ ${ }^{1}$ Faculty of Nursing, Baqiyatallah University of Medical Sciences, Tehran, IR Iran \\ ${ }^{2}$ Behavioral Sciences Research Center, Nursing Faculty, Baqiyatallah University of Medical Sciences, Tehran, IR Iran \\ "Corresponding author: Hosien Babatabar Darzi, Faculty of Nursing, Baqiyatallah University of Medical Sciences, Tehran, IR Iran. E-mail: babatabar1343@yahoo.com
}

Received 2016 December 26; Revised 2017 February 28; Accepted 2017 March 05.

\begin{abstract}
Background: Complementary therapies are cost-effective and non-invasive methods, aimed at increasing comfort in stressful situations. The aim of this study was to compare the effects of aromatherapy with rose and lavender fragrances on the physiological parameters of patients undergoing open heart surgery.

Methods: This randomized, controlled, clinical study was performed on 160 patients, undergoing open heart surgery in 2015 . The patients were randomly allocated to 4 groups: 2 intervention groups, 1 control group, and 1 placebo group. After transferring the patients from the operating room to the cardiovascular intensive care unit (ICU), the vital signs were documented in all the groups with the patient's first inspiratory effort, using a monitoring device. In the intervention group, after the patient's first inspiratory effort, a cotton cloth, impregnated with 3 drops of rose and lavender fragrances, was attached to the patient's endotracheal tube. In the placebo group, intervention was performed with a cotton cloth impregnated with water. In the control group, no intervention was applied. Afterwards, the patients' vital signs were controlled and documented every 30 minutes until the endotracheal tube was extracted. Following endotracheal tube extraction, the vital signs were documented every 15 minutes for 1 hour. For data analysis, descriptive and inferential statistics were calculated, using SPSS version 23.

Results: The results of the present study indicated that aromatherapy with rose and lavender fragrances does not cause any significant difference in the physiological indicators. The decline in systolic blood pressure within the first 15, 45, and 60 minutes following endotracheal tube extraction was significantly different among the groups. However, although the difference was statistically significant, it was not of clinical importance.

Conclusions: Considering the positive effects of aromatherapy on hemodynamic indicators, this method can be applied as an effective complementary treatment.
\end{abstract}

Keywords: Aromatherapy, Physiological Parameters, Open Heart Surgery

\section{Background}

Today, cardiovascular diseases are the most prevalent, chronic, and fatal conditions in the world (1), accounting for more than 16 million deaths worldwide. More than $80 \%$ of deaths in low- and middle-income countries are caused by cardiovascular diseases (2). There are different methods of treatment for these patients, including surgery, which is one of the most common medical interventions (3). Almost 68600 open heart surgeries are performed in the United States everyyear, and above 20000 open heart surgeries are annually carried out in Australia (4).

Patients undergoing coronary artery bypass grafting (CABG) are transferred to intensive care units (ICUs) where they are normally under mechanical ventilation during the first 6 hours following surgery )fast-track extubation) (5). Anxiety and agitation in these patients result in sleep disorders, increased oxygen consumption by the heart muscles, increased burden of the sympathetic nervous system, tachypnea, increased heart rate, neural-hormonal responses, and increased blood pressure, which challenge the process of weaning from mechanical ventilation.

Changes in vital signs, increased oxygen demand of the heart, and increased mortality rate are observed following anxiety in patients suffering from cardiac diseases $(6,7)$. Also, medication therapies are often associated with complications, such as hypotension, reduced vital functions (such as reduced breathing and heart rate), drowsiness, nausea and vomiting, constipation, allergic reactions (in some cases), and even shock. Besides several physical and psychological complications, drug dependence, and drug tolerance among patients, these therapies also lead to high healthcare costs for the healthcare systems (8).

On the other hand, anti-anxiety drugs can cause delay in the process of weaning from ventilators (9). Use of non- 
pharmacological methods has been emphasized in recent years for reducing pain and anxiety. Non-pharmacological methods constitute a wide range of treatments, which are relatively more simple, cost-effective, non-invasive, and complication-free in comparison with pharmacological drugs (10).

Aromatherapy, which is based on the use of plant extracts, is specifically applied in complementary medicine. Volatile oil essences, which consist of special compounds, are used for wound healing, mood boost, anxiety relief, relaxation, and pain relief (11). Lavender fragrance is one of the common fragrances, used as a sedative in aromatherapy. Lavandula angustifolia is the scientific term of lavender, and linalool and linalyl acetate are among the active ingredients. Linalool acts as a sedative by influencing gamma-aminobutyric acid receptors in the central nervous system, while lynalyl acetate has a narcotic function (12).

On the other hand, rose fragrance can influence the central nervous system with 2 constituents, ie, citronellol and phenethyl alcohol, which produce anti-anxiety effects (13). There are several studies regarding the effects of aromatherapy on anxiety, pain, vital signs, sleep quality, nausea, and stress (14-19). In this regard, Jung et al. showed that ylang-ylang fragrance can remarkably influence systolic and diastolic blood pressure and decrease heart rate in male athletes (20). Also, Chung et al. revealed that exposure to essential oils for less than 1 hour significantly reduces workers' heart rate and blood pressure (21). On the other hand, Peng et al. (2009) showed that aromatherapy with bergamot does not have any significant effects on decreasing blood pressure or heart rate (22).

There is a scarcity of information regarding the effects of aromatherapy on the physiological parameters of open heart surgery patients, and contradictory results have been reported in studies applying these methods in the medical system. Therefore, the aim of this study was to compare the effects of aromatherapy with rose and lavender fragrances on the physiological parameters of patients undergoing open heart surgery. This study can be an important step in understanding aromatherapy and can provide scientific results in this area.

\section{Methods}

This randomized, controlled, clinical trial was performed among 4 groups during January-March 2016 at the cardiovascular ICU of Baqiyatallah Teaching hospital, affiliated to Baqiyatallah University of Medical Sciences, Tehran, Iran. The study population included patients hospitalized in the cardiovascular ICU under mechanical ventilation after open heart surgery.
The inclusion criteria were as follows: (1) candidacy for open heart surgery (eg, patients with coronary artery problems, congenital disorders, and reconstructive valve surgery); (2) lack of olfactory problems; (3) age $>18$ years and $<70$ years; (4) heart rate $>60 \mathrm{bpm}$; (5) systolic blood pressure > $90 \mathrm{mmHg}$; (6) no prior experience of cardiac surgery; (7) undergoing non-emergency open heart surgery; (8) no clotting disorders (international normalized ratio $<2.5$ ); and (9) lack of sensitivity to the fragrances.

On the other hand, the exclusion criteria were as follows: (1) administration of more than 1 inotrope; (2) return to the operating room; (3) unstable hemodynamics; and (4) loss of consciousness. The sample size was set at 40 cases for each group, based on the Altman's nomogram (type I error, 0.05; power, 80\%), similar to a study by Ebadi et al. (2015) (23).

The night before surgery, the patients were randomly allocated to one of the following groups: aromatherapy with lavender fragrance, aromatherapy with rose fragrance, placebo, and control. A total of 40 samples were assigned to each group. In the intervention groups, after transferring the patient from the operating room to the cardiovascular ICU, a cotton cloth, impregnated with 3 drops of rose or lavender fragrance, was attached to the patient's tracheal tube after the first inspiratory effort. In the placebo group, intervention was carried out with a cotton cloth, impregnated with water. The control group received no intervention.

The vital signs were controlled and documented every 30 minutes until the endotracheal tube was extracted. Following tracheal tube extraction, the vital signs were assessed every 15 minutes. The patients' demographic information was collected using a questionnaire, including age, gender, body mass index (BMI), ventricular ejection fraction, blood pump time, history of diabetes mellitus, smoking, blood pressure, and pulmonary diseases. The demographic information was gathered through interviews and medical records.

Physiological indicators included heart rate, respiratory rate, systolic and diastolic blood pressure, and percentage of peripheral oxygen saturation, which were measured using a vital sign monitoring system and a monitoring device (Datks, General Electric Co., USA). For determining the amount of the required fragrance, the patients' opinions were surveyed in a pilot study before the interventions. It was found that use of 1 drop is insensible for some patients, while use of 5 drops is excessive and inappropriate for others; therefore, 3 drops were used as the moderate amount. Rose and lavender fragrances were prepared by Kashan Barich Essence Company (100\% pure concentration). A cotton cloth impregnated with the fragrances and water was attached to the patients since the 
patient's first inspiratory effort for extubation.

Permission was obtained from the ethics committee of the university (IR.Bmsu.Rec.1394.141), and the study was registered in the Iranian registry of clinical trials (IRCT201510012730N9). The authorities were coordinated with, the aim of the study was explained to the patients, and written informed consents regarding participation in the study were obtained. The patients were assured about the confidentiality of the information, and they were allowed to withdraw from the study at any time. After data collection, statistical analysis was performed, using SPSS version 23.

\section{Results}

The participants in this study were within the age range of 35 - 70 years (average, 58.59 years). Also, most of the participants were male (60.6\%). There was no significant difference between the groups in terms of demographic parameters $(\mathrm{P}>0.05)$, indicating that they were homogeneous before the intervention.

Chi square and ANOVA tests were used for assessing the statistical information. The results revealed that aromatherapy did not cause any significant changes in the physiological indicators (Figures 1 - 4). Systolic blood pressure was significantly different among the groups within the first 15, 45, and 60 minutes following tracheal tube extraction. The results showed that aromatherapy with rose and lavender fragrances is effective in stability and decreasing systolic blood pressure (Figure 5).

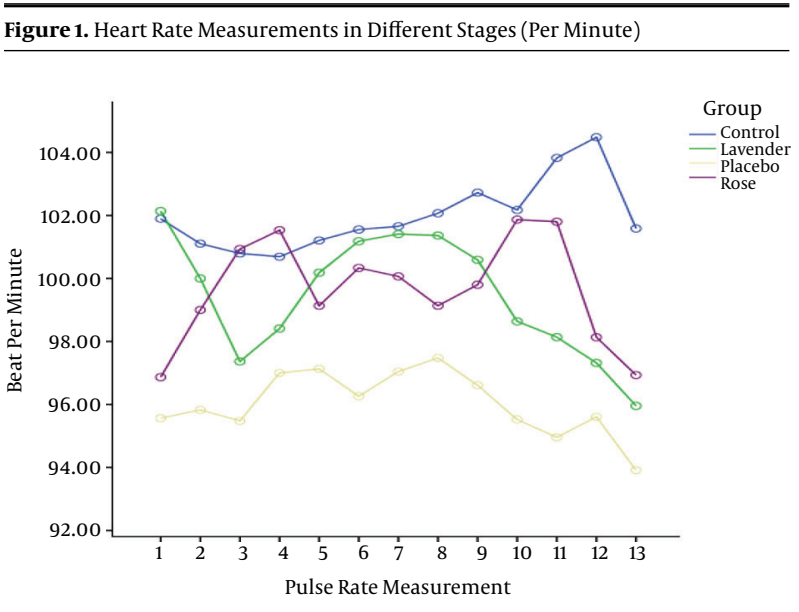

Comparison of changes in the mean and standard deviation of heart rate in different stages among the 4 groups. Repeated measures ANOVA showed no significant difference between the groups in terms of changes in heart rate $(\mathrm{P}=0.33)$.

According to Tukey post hoc test results, there was a significant difference within the first 15 minutes between the

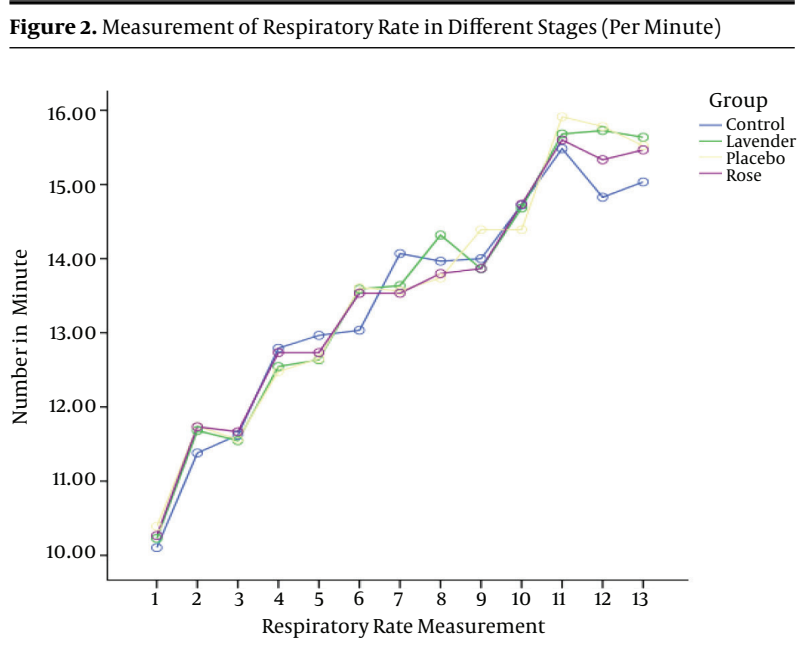

Comparison of changes in the mean and standard deviation of respiratory rate in different stages among the 4 groups Repeated measures ANOVA showed no significant difference between the 4 groups in terms of changes in respiratory rate $(\mathrm{P}=$ $0.11)$

Figure 3. Diastolic Blood Pressure Measurements in Different Stages (mmHg)

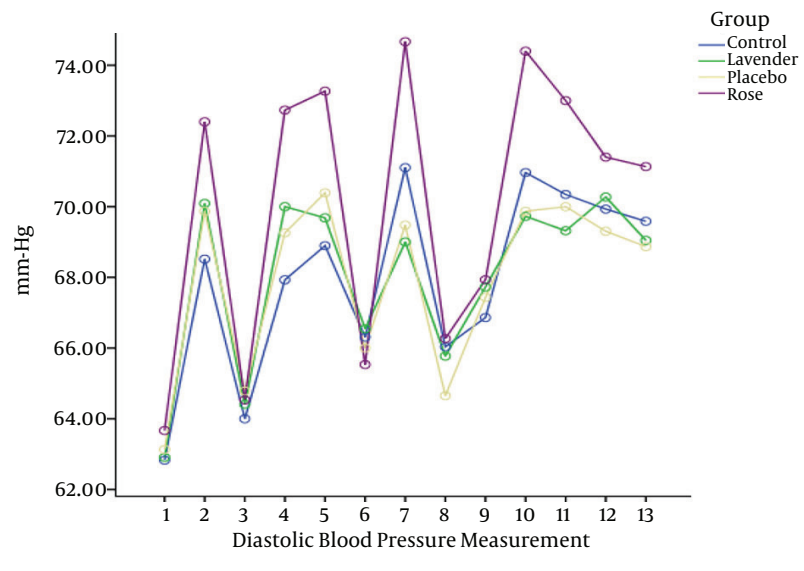

Comparison of changes in the mean and standard deviation of diastolic blood pressure in different stages among the 4 groups. Repeated measures ANOVA showed no significant difference between the 4 groups in terms of diastolic blood pressure ( $\mathrm{P}$ $=0.94)$.

rose fragrance and control groups, while during the first 45 and 60 minutes following tracheal tube extraction, there was a significant difference between the rose fragrance and placebo groups. Although the difference was statistically significant, it had no clinical importance.

Increased systolic blood pressure in the control and placebo groups could be related to extubation, and decreased blood pressure in the rose fragrance group could be attributed to the sedative and analgesic effects of rose fragrance (24), although it was not clinically effective. For assessing the statistical data among the 4 groups in 13 
Figure 4. Measurement of Peripheral Oxygen Saturation (Percentage)

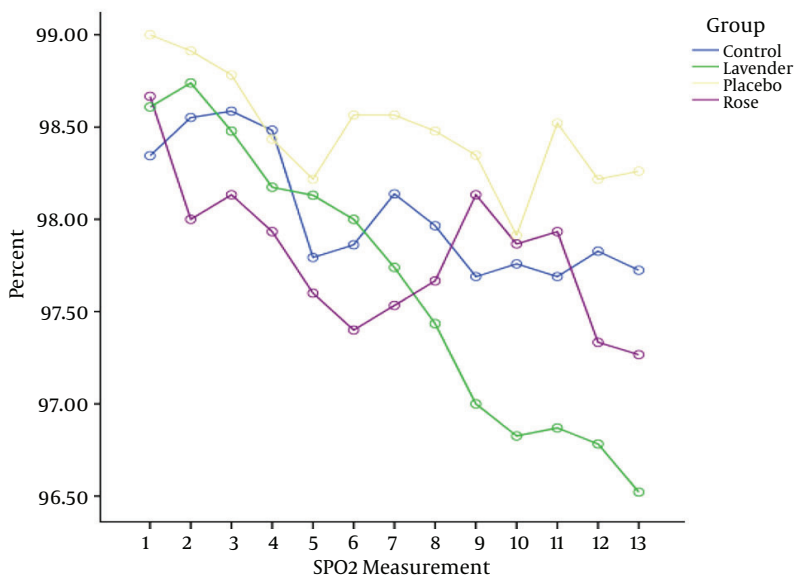

Comparison of changes in the mean and standard deviation in different stages among the 4 groups. Repeated measures ANOVA showed no significant difference between the 4 groups in terms of oxygen saturation percentage $(P=0.05)$.

Figure 5. Systolic Blood Pressure Measurements in Different Stages (mmHg)

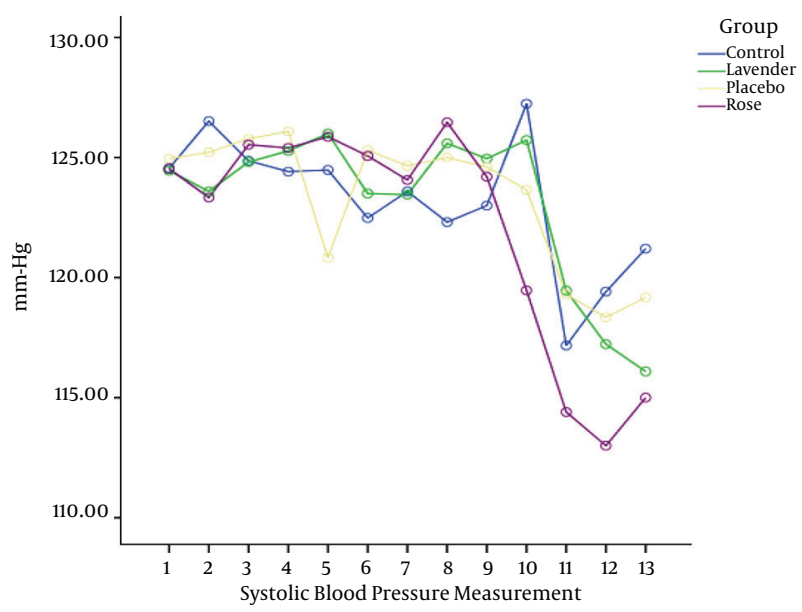

Comparison of changes in the mean and standard deviation of systolic blood pressure in different stages among the 4 groups. Repeated measures ANOVA showed no significant difference between the 4 groups in terms of systolic blood pressure $(\mathrm{P}=$ $0.30)$.

measurement stages, repeated measures ANOVA and Tukey post hoc test were used.

\section{Discussion}

The aim of the present study was to assess the effect of inhalation aromatherapy with lavender and rose fragrances on the physiological parameters of patients undergoing open heart surgery. The findings indicated no significant difference between the groups after the intervention. Overall, several studies have been conducted regarding the effects of inhalation aromatherapy on patients undergoing open heart surgery.

In this regard, Nategh et al. (2015) assessed the effects of aromatherapy with lavender on hemodynamic indicators among acute coronary syndrome patients. Lytel et al. (2014) evaluated the effects of aromatherapy with lavender on vital signs and sleep quality among ICU-admitted patients. Mi Cho et al. (2012) assessed the effects of inhaling lavender and thyme on blood pressure, heart rate, sleep, stress, and anxiety among patients with basic hypertension. In consistence with the present study, the results indicated that aromatherapy does not have any significant effects on physiological parameters. It should be noted that in studies by Nategh et al. Lytel et al. and Mi Choi et al. aromatherapy duration was 20 minutes, 8 hours, and 3 times per week during 2 weeks, respectively (25-27).

In addition, Aghagoli et al. (2016) assessed the effects of aromatherapy with rose fragrance on apnea, bradycardia, and arterial blood oxygen in preterm infants. Also, Cho et al. (2013) evaluated the effects of aromatherapy with lavender, Citrus aurantium, and chamomile on anxiety, vital signs, and sleep quality of percutaneous coronary intervention (PCI) patients in an ICU setting. In addition, Kim et al. (2010) studied the effects of aromatherapy with lavender, chamomile, and sweet orange on blood pressure and pulse rate, using VAS and McNair scales among nursing students performing intravenous injection for the first time.

Also, Hsin Hu et al. (2010) evaluated the effects of aromatherapy with Citrus aurantium on decreasing anxiety, stress, and physiological parameters in patients undergoing colonoscopy $(19,28-30)$. The results of the 4 mentioned studies showed that aromatherapy has significant effects on physiological parameters, which is inconsistent with the present study. This discrepancy may be attributed to differences in the study samples, applied methods, type of fragrances, and aromatherapy duration.

In the study by Aghagoli et al. the results showed that apnea, bradycardia, and decreased arterial blood oxygen were less prevalent in the intervention group, compared to the control group. Furthermore, in studies by Hsin Hu et al. and Kim et al. there was a significant decline in systolic blood pressure and heart rate. In addition, in the study by Cho et al. there was a remarkable decline in systolic and diastolic blood pressure.

Overall, various studies have evaluated the effects of complementary medicine. In this regard, Ebadi (2015) assessed the effects of foot reflexology massage on the physiological parameters and time of weaning from mechanical ventilators among patients undergoing open heart surgery. Based on the findings, foot reflexology massage 
decreased the time of weaning from ventilator, whereas it did not influence the physiological parameters (24). Also, Bagheri studied the effects of foot reflexology massage on anxiety following $\mathrm{CABG}$, which resulted in decreased anxiety (4).

\section{Acknowledgments}

The present study was part of a thesis project, submitted to Baqiyatallah University of Medical Sciences. We would like to thank from the "clinical research development center of Baqiyatallah hospital " for their kindly cooperation, and Mr. Zalpoli, the head nurse of cardiovascular ICU of Baqiyatallah Hospital. This study was funded by the Clinical Research Development Center of Baqiyatallah Hospital. The authors would like to thank the participants for their sincere cooperation in this study.

\section{Footnotes}

Authors' Contribution: Saeed Ghasemi: study conception and design, data collection and analysis, and drafting of the manuscript. Hosein Babatabar: study conception and design, critical revisions for important intellectual content, drafting of the manuscript, and supervision. Abbas Ebadi: substantial contributions to analysis and interpretation of data, critical revisions for important intellectual content, and approval of the final version of the manuscript.

Conflict of Interests: The authors declare no conflicts of interest with respect to the authorship and/or publication of this article.

\section{References}

1. Sheikhi MA, Ebadi A, Gholizadeh B, Ramezani A. Risk factors for hemodynamics change after cabg surgery in southwest of Iran. Int J Bioassays. 2015;4(02):3659-63.

2. Mohammadi E. The perception of cardiac surgery patients on comfortable resources: a qualitative study. J Qual Res Health Sci. 2012;1(2):123-34.

3. Kavei P, Ebadi A, Moradian ST, Rahimabadi MS. The Effect of Massage Therapy on Psychological Outcomes in Patients after Cardiac Surgery: A Mini Review. Int J Med Rev. 2015;1(4).

4. Bagheri-Nesami M, Shorofi SA, Zargar N, Sohrabi M, GholipourBaradari A, Khalilian A. The effects of foot reflexology massage on anxiety in patients following coronary artery bypass graft surgery: a randomized controlled trial. Complement Ther Clin Pract. 2014;20(1):42-7. doi: 10.1016/j.ctcp.2013.10.006. [PubMed: 24439644].

5. Nouri JM, Sohrabi B, Moradian ST, Ghiasi SMS. Effect of Adaptive Support Ventilation Weaning Mode in Two Conventional or Standard Methods on Respiratory and Hemodynamic Performance Indices: A Randomized Clinical Trial.Trauma Mon. 2016.
6. Adib-Hajbaghery M, Rajabi-Beheshtabad R, Abasi A, Azizi-Fini E. The effect of massage therapy by a nurse and the patient's companion on the anxiety of male patients hospitalized in CCU: a clinical trial. Iran J Nurs. 2012;25(78):72-83.

7. Chlan LL. Description of anxiety levels by individual differences and clinical factors in patients receiving mechanical ventilatory support. Heart Lung. 2003;32(4):275-82. [PubMed: 12891169].

8. Twiss E, Seaver J, McCaffrey R. The effect of music listening on older adults undergoing cardiovascular surgery. Nurs Crit Care. 2006;11(5):224-31. [PubMed: 16983853].

9. Lee OK, Chung YF, Chan MF, Chan WM. Music and its effect on the physiological responses and anxiety levels of patients receiving mechanical ventilation: a pilot study. J Clin Nurs. 2005;14(5):609-20. doi: 10.1111/j.1365-2702.2004.01103.x. [PubMed:15840076].

10. Zakerimoghadam M, Shaban M, Mehran A, Hashemi S. Effect of muscle relaxation on anxiety of patients undergo cardiac catheterization. J Hayat. 2010;16(2):64-71.

11. Heidari T, Roozbahani N, Attarha M, Akbari Torkestani N, Bekhradi R, Siyanaki V. Effect of aromatherapy on pain severity in primary dysmenorrhea. Arak Med Univ J. 2012;15(4):1-9.

12. Najafi Z, Taghadosi M, Sharifi K, Farrokhian A, Tagharrobi Z. The effects of inhalation aromatherapy on anxiety in patients with myocardial infarction: a randomized clinical trial. Iran Red Crescent Med J. 2014;16(8):e15485. doi: 10.5812/ircmj.15485. [PubMed: 25389481].

13. Umezu T, Ito H, Nagano K, Yamakoshi M, Oouchi H, Sakaniwa M, et al. Anticonflict effects of rose oil and identification of its active constituents. Life Sci. 2002;72(1):91-102. [PubMed: 12409148].

14. Hunt R, Dienemann J, Norton HJ, Hartley W, Hudgens A, Stern $\mathrm{T}$, et al. Aromatherapy as treatment for postoperative nausea: a randomized trial. Anesth Analg. 2013;117(3):597-604. doi: 10.1213/ANE.0b013e31824a0b1c. [PubMed: 22392970].

15. Ju MS, Lee S, Bae I, Hur MH, Seong K, Lee MS. Effects of aroma massage on home blood pressure, ambulatory blood pressure, and sleep quality in middle-aged women with hypertension. Evid Based Complement Alternat Med. 2013;2013:403251. doi: 10.1155/2013/403251. [PubMed: 23431338].

16. Gelinas C, Arbour C, Michaud C, Robar L, Cote J. Patients and ICU nurses' perspectives of non-pharmacological interventions for pain management. Nurs Crit Care. 2013;18(6):307-18. doi: 10.1111/j.14785153.2012.00531.x. [PubMed: 24165072].

17. Atashi N, Bahari SM, Sanatkaran A. The effects of Red rose essential oil aromatherapy on athletes' sleep quality before the competition. J Novel Appl Sci. 2015.

18. Metawie MAH, Amasha HAR, Abdraboo RA, Ali SE. Effectiveness of Aromatherapy with Lavender Oil in Relieving Post Caesarean Incision Pain.J Surg. 2015;3(2-1):8-13.

19. Aghagoli S, Salimi A, Salimi M, Ghazavi Z, Marofi M, Mohammadbeigi A. Aromatherapy with Rosa Damascenes in Apnea, Bradycardia and Spo2 of Preterm Infants; a Randomized Clinical Trial. Int J Pediatr. 2016;4(6):1911-8.

20. Jung DJ, Cha JY, Kim SE, Ko IG, Jee YS. Effects of Ylang-Ylang aroma on blood pressure and heart rate in healthy men. J Exerc Rehabil. 2013;9(2):250-5. doi: 10.12965/jer.130007. [PubMed: 24278868].

21. Chuang KJ, Chen HW, Liu IJ, Chuang HC, Lin LY. The effect of essential oil on heart rate and blood pressure among solus por aqua workers. Eur J Prev Cardiol. 2014;21(7):823-8. doi: 10.1177/2047487312469474. [PubMed: 23197402].

22. Peng SM, Koo M, Yu ZR. Effects of music and essential oil inhalation on cardiac autonomic balance in healthy individuals. J Altern Complement Med. 2009;15(1):53-7. doi: 10.1089/acm.2008.0243. [PubMed: 19769477].

23. Ebadi A, Kavei P, Moradian ST, Saeid Y. The effect of foot reflexology on physiologic parameters and mechanical ventilation weaning time in patients undergoing open-heart surgery: A clinical trial study. Complement Ther Clin Pract. 2015;21(3):188-92. doi: 10.1016/j.ctcp.2015.07.001. [PubMed: 26256138]. 
24. Reza E, Nezhad H, Kamyab S. Pharmacological Effects of Rosa Damascena* Mohammad Hossein Boskabady, Mohammad Naser Shafei, Zahra Saberi, 2 Somayeh Amini. ; 2010.

25. Nategh M, Heidari MR, Ebadi A, Kazemnejad A, Babaei Beigi MA. Effect of lavender aromatherapy on hemodynamic indices among patients with acute coronary syndrome: a randomized clinical trial.J Crit Care Nurs. 2015;7(4):201-8.

26. Lytle J, Mwatha C, Davis KK. Effect of lavender aromatherapy on vital signs and perceived quality of sleep in the intermediate care unit: a pilot study. Am J Crit Care. 2014;23(1):24-9. doi: 10.4037/ajcc2014958. [PubMed: 24382614].

27. Choi EM, Lee KS. Effects of Aroma inhalation on Blood Pressure, Pulse Rate, Sleep, Stress, and Anxiety in Patients with Essential Hypertension. J Korean Biol Nurs Sci. 2012;14(1):41-8. doi: 10.7586/jkbns.2012.14.1.41.

28. Cho MY, Min ES, Hur MH, Lee MS. Effects of aromatherapy on the anxiety, vital signs, and sleep quality of percutaneous coronary intervention patients in intensive care units. Evid Based Complement Alternat Med. 2013;2013:381381. doi: 10.1155/2013/381381. [PubMed: 23476690].

29. Kim M, Hwangbo HH. Randomized trial evaluating the aroma inhalation on physiological and subjective anxiety indicators of the nursing students experiencing the first intravenous injection. Int J Bio Sci Bio Technol. 2010;2(4):1-9.

30. Hu PH, Peng YC, Lin YT, Chang CS, Ou MC. Aromatherapy for reducing colonoscopy related procedural anxiety and physiological parameters: a randomized controlled study. Hepatogastroenterology. 2010;57(102-103):1082-6. [PubMed: 21410035]. 